\title{
Closed-Form Estimations of the Bistable Region in Metal Cutting via the Method of Averaging
}

\author{
Tamas G. Molnar ${ }^{\mathrm{a}, *}$, Tamas Insperger ${ }^{\mathrm{b}}$, Gabor Stepan ${ }^{\mathrm{a}}$ \\ ${ }^{a}$ Department of Applied Mechanics, Budapest University of Technology and Economics, H-1111 Budapest, Hungary \\ ${ }^{b}$ Department of Applied Mechanics, Budapest University of Technology and Economics and MTA-BME Lendület Human Balancing Research Group, H-1111 \\ Budapest, Hungary
}

\begin{abstract}
Machine tool vibrations in turning processes are analyzed by taking into account the nonlinearity of the cutting force characteristics. Unstable limit cycles are computed for the governing nonlinear delay-differential equation in order to determine the bistable technological parameter region where stable stationary cutting and large-amplitude machine tool vibrations coexist. Simple closedform formulas are derived for the amplitude of limit cycles and for the size of the bistable region considering a general cutting force characteristics. The analytical results are determined by the method of averaging, which can be used to treat the nonlinearities without their third-order approximation. The results are confirmed by numerical continuation and using Melnikov's integral.
\end{abstract}

Keywords: machine tool chatter, nonlinear dynamics, bistability, averaging

\section{Introduction}

Understanding the dynamics of harmful machine tool vibrations (chatter) during metal cutting has been of interest since the 1950's. Chatter produces noise, reduces the quality of the machined surface, increases tool wear, and may even damage the machine tool and/or the workpiece, hence it must be avoided or suppressed. According to the works of Tobias [1] and Tlusty [2], one main source of machine tool vibrations is the so-called surface regeneration effect: the vibrations of the tool are copied onto the machined surface during metal cutting, which then excite the vibrations in the subsequent cut. This leads to the regeneration of the waviness of the machined surface during the consecutive cuts.

Since the actual vibrations depend on the vibrations recorded on the surface at the previous cut, the dynamics of machine tool chatter is described by delay-differential equations (DDEs). Turning or drilling processes are typically described by autonomous DDEs, while milling can be modeled by timeperiodic DDEs $[3,4,5,6,7,8,9,10]$. In what follows, we restrict ourselves to autonomous equations. The DDEs governing machine tool chatter are typically nonlinear, since the cutting force is a nonlinear function of the chip thickness [7].

Machine tool vibrations can be explained by the instability of the stationary solution (equilibrium) of the nonlinear (autonomous) DDE. The (in)stability is conventionally illustrated in stability lobe diagrams. These are depicted in the plane of the most important technological parameters - the spindle speed

\footnotetext{
${ }^{*}$ Corresponding author

Email addresses: molnar@mm. bme.hu (Tamas G. Molnar), insperger@mm. bme.hu (Tamas Insperger), stepan@mm. bme.hu (Gabor Stepan)
}

and the depth or the width of cut - and distinguish the regions associated with stable machining from those of machine tool chatter. When chatter occurs, the stability of stationary cutting (the equilibrium) is lost via Hopf bifurcation. According to $[11,12,13,14]$, the Hopf bifurcation is typically subcritical and gives rise to an unstable periodic orbit (limit cycle) in the vicinity of the linearly stable equilibrium. Therefore, there exists a region of bistability $[15,14,16,17,18,19]$ where the basin of attraction of the linearly stable equilibrium does not cover the whole phase space and chatter occurs to large enough perturbations. Here, stable stationary cutting coexists with large-amplitude chatter, hence this region is unsafe and preferably must be avoided.

In this paper, we focus on computing the limit cycle and the bistable technological parameter region for turning processes. Since the phase space of DDEs is infinite dimensional [20], such analysis is nontrivial, although there exist several analytical approaches to compute limit cycles for nonlinear DDEs. The two most popular methods are center manifold reduction [21, 22, 23, 24, 25] and the method of multiple scales [26], but there exist several other approaches such as the method of small parameters [26, 27], harmonic balance [16, 28], or Melnikov's integral [29]. For orthogonal cutting models, center manifold reduction is discussed in $[11,12,13,14]$, while the method of multiple scales is presented in [30, 31, 32]. These analyses are typically restricted to a cubic approximation of the nonlinearity, since higher-order terms lead to extremely long and difficult expressions.

In this paper, we use the method of averaging [33, 34, 35, $36,37,38,39,40,41]$ to compute the periodic orbit. This method can easily be employed for higher-order nonlinearities, however, the analysis involves less rigorous derivations than the center manifold reduction or the method of multiple scales. The 


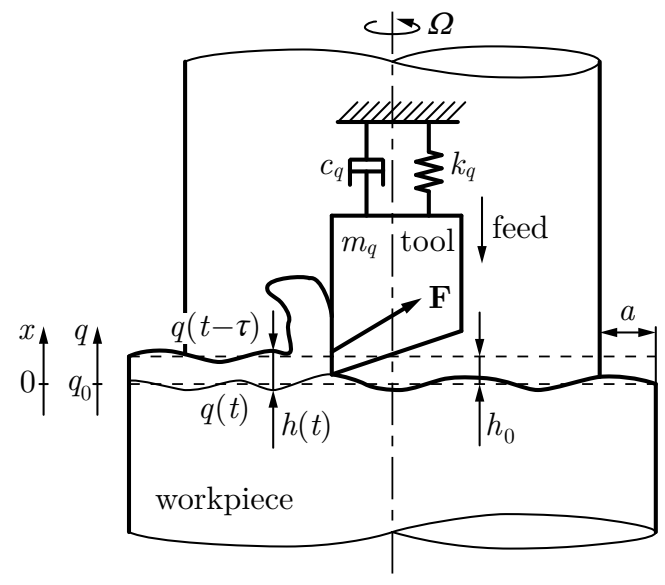

Figure 1: Single-degree-of-freedom mechanical model of turning.

results obtained below are valid only if the periodic solution of the nonlinear DDE is close to harmonic. We show that this condition is satisfied for the most typical cutting force expressions. Note that extensions of the averaging method to use explicitly given non-harmonic periodic expressions also exist, see for example [29, 42]. We also demonstrate that the same analysis could be conducted alternatively by Melnikov's integral, and we verify the analytical results by numerical continuation.

The rest of the paper is organized as follows. The mechanical model of turning processes with the governing DDE is presented in Sec. 2. Linear stability analysis and the phenomenon of bistability are reviewed in Sec. 3 based on the existing literature. The method of averaging is used in Sec. 4 to derive simple approximate analytical formulas for the amplitude of periodic solutions in Sec. 5 and for the size of the bistable region in Sec. 6. Discussion and comparison to other approaches are presented in Sec. 7.

\section{Mechanical Model}

Consider the mechanical model of turning processes shown in Fig. 1. We assume that the workpiece is rigid, the tool is compliant and its vibrations can be described by a single dominant vibration mode that takes place along the feed direction. The equation governing the tool's motion is

$$
\ddot{q}(t)+2 \zeta \omega_{\mathrm{n}} \dot{q}(t)+\omega_{\mathrm{n}}^{2} q(t)=\frac{1}{m_{q}} F_{q}(h(t), a),
$$

where $m_{q}$ is the modal mass, $\omega_{\mathrm{n}}$ is the undamped natural angular frequency and $\zeta$ is the damping ratio of the dominant vibration mode described by the generalized coordinate $q$.

$F_{q}$ denotes the generalized force that is the $q$-directional component of the cutting force acting on the tool. It is typically proportional to the chip width $a$ and depends on the uncut chip thickness $h$ [7], see Fig. 2(a). This is expressed by

$$
F_{q}(h, a)= \begin{cases}a f_{q}(h) & \text { if } h>0, \\ 0 & \text { if } h \leq 0,\end{cases}
$$

(a)

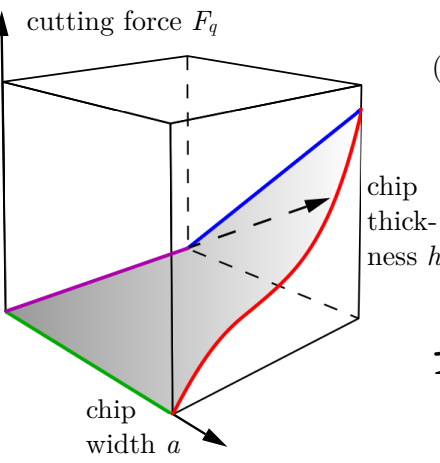

(b) $\quad h(t)>0$

(c)
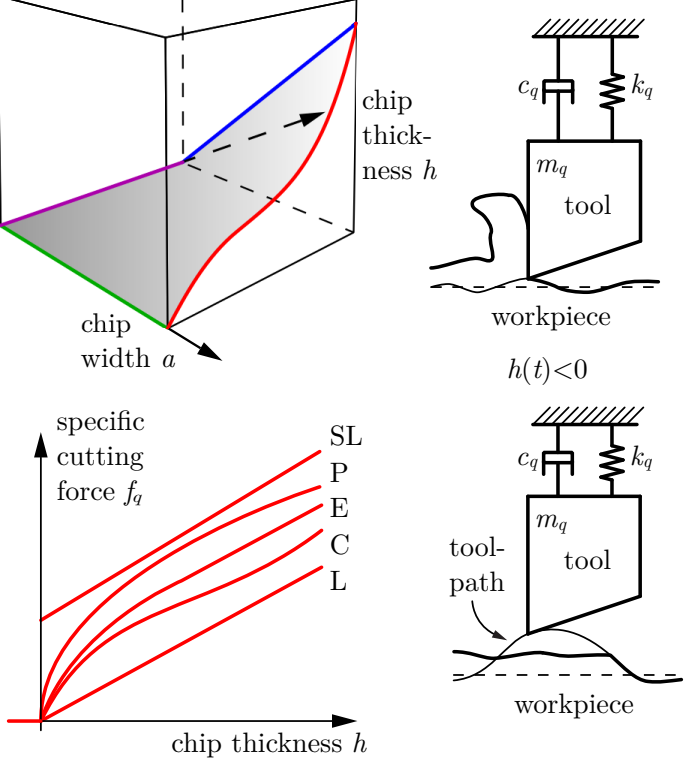

Figure 2: (a) Characteristics (2) of the cutting force; (b) illustration of the flyover effect; (c) models (3)-(7) of the specific cutting force [43].

where the specific cutting force $f_{q}(h)$ is a function of the uncut chip thickness $h$. The case $h \leq 0$ occurs if the tool jumps out of the workpiece and loses contact with the material due to largeamplitude vibrations. In such cases, the cutting force becomes zero. This phenomenon is called flyover and is illustrated in Fig. 2(b). In what follows, we restrict ourselves to cases where the tool remains in contact with the workpiece during machining.

The relationship between the specific cutting force $f_{q}$ and the uncut chip thickness $h$ can be described by various models, see [43] and the references therein. The most widely accepted expressions are the linear (L) and shifted linear (SL) functions [7], the power law (P) [44, 7], the cubic polynomial (C) [15] and the exponential (E) cutting force characteristics [45] that are given by

$$
\begin{aligned}
& f_{q}^{\mathrm{L}}(h)=K_{\mathrm{c}} h, \\
& f_{q}^{\mathrm{SL}}(h)=K_{\mathrm{e}}+K_{\mathrm{c}} h, \\
& f_{q}^{\mathrm{P}}(h)=K_{v} h^{v}, \\
& f_{q}^{\mathrm{C}}(h)=\rho_{1} h+\rho_{2} h^{2}+\rho_{3} h^{3}, \\
& f_{q}^{\mathrm{E}}(h)=b_{1} h+\frac{b_{2}}{b_{3}} \mathrm{e}^{b_{3} h}+b_{4},
\end{aligned}
$$

respectively. Here, $K_{\mathrm{e}, \mathrm{c}}, K_{v}, v, \rho_{1,2,3}, b_{1,2,3,4}$ are parameters that can be obtained from experimental cutting force measurements. The specific cutting force models (3)-(7) are illustrated in Fig. 2(c). Now, we focus on the nonlinear expressions: we analyze the power law (5), the cubic polynomial (6) and the exponential function (7) in detail.

According to the theory of regenerative machine tool chatter $[1,2]$, the instantaneous uncut chip thickness $h(t)$ is deter- 
mined by the actual tool position $q(t)$ and the position $q(t-\tau)$ of the previous cut taking place one workpiece revolution before. Therefore,

$$
h(t)=h_{0}+q(t-\tau)-q(t),
$$

where $h_{0}$ is the prescribed chip thickness (feed per revolution). Parameter $\tau$ is called the regenerative delay. It is equal to the rotational period and is related to the angular velocity $\Omega$ of the workpiece by $\tau=2 \pi / \Omega$.

Equations (1), (2) and (8) form a nonlinear delay-differential equation. This equation has a unique equilibrium $q(t) \equiv q_{0}$ that can be given in the form

$$
q_{0}=\frac{a f_{q}\left(h_{0}\right)}{m_{q} \omega_{\mathrm{n}}^{2}} .
$$

The equilibrium describes the ideal (chatter-free) machining: stationary cutting with constant prescribed chip thickness $h(t) \equiv h_{0}$. The onset of machine tool chatter is associated with the instability of the equilibrium. In order to analyze stability, we introduce the dimensionless coordinate

$$
x(t)=\frac{q(t)-q_{0}}{h_{0}} .
$$

We also use the dimensionless time $\tilde{t}=\omega_{\mathrm{n}} t$ and the dimensionless delay $\tilde{\tau}=\omega_{\mathrm{n}} \tau$. The derivative with respect to $\tilde{t}$ is indicated by prime and satisfies $\dot{x}(t)=\omega_{\mathrm{n}} x^{\prime}(\tilde{t})$. After dropping the tildes, we obtain

$$
x^{\prime \prime}(t)+2 \zeta x^{\prime}(t)+x(t)=\frac{a}{m_{q} \omega_{\mathrm{n}}^{2}} \frac{f_{q}(h(t))-f_{q}\left(h_{0}\right)}{h_{0}},
$$

where $h(t)$ is given by Eq. (8) and can be expressed using coordinate $x$ via Eq. (10).

Now, we expand $f_{q}(h(t))$ into Taylor series around $h_{0}$ :

$$
f_{q}(h(t))=\sum_{m=0}^{\infty} \frac{1}{m !} f_{q}^{(m)}\left(h_{0}\right) h_{0}^{m}(x(t-\tau)-x(t))^{m} .
$$

Substitution of Eq. (12) into Eq. (11) yields the dimensionless equation of motion in the form

$$
x^{\prime \prime}(t)+2 \zeta x^{\prime}(t)+x(t)=w \sum_{m=1}^{\infty} \eta_{m}(x(t-\tau)-x(t))^{m},
$$

where the expressions of the dimensionless chip width $w$ (that is proportional to the actual chip width $a$ ) and the dimensionless cutting force coefficients $\eta_{m}$ read

$$
\begin{aligned}
w & =\frac{a f_{q}^{\prime}\left(h_{0}\right)}{m_{q} \omega_{\mathrm{n}}^{2}}, \\
\eta_{m} & =\frac{1}{m !} \frac{f_{q}^{(m)}\left(h_{0}\right)}{f_{q}^{\prime}\left(h_{0}\right)} h_{0}^{m-1}, \quad m \in \mathbb{Z}^{+} .
\end{aligned}
$$

Note that $\eta_{1}=1$.

\section{Linear Stability and Bistability}

Via linear stability analysis [46], it can be shown that the machining is stable for chip widths $0<w<w_{\mathrm{H}}$. The linear stability boundaries can be obtained via D-subdivision [46] by substituting the trial solution $x(t)=X \mathrm{e}^{\mathrm{i} \omega t}, X \in \mathbb{C} \backslash\{0\}$ into the linear part of Eq. (13), separating real and imaginary parts, and solving

$$
\begin{aligned}
-\omega^{2}+1+w(1-\cos (\omega \tau)) & =0, \\
2 \zeta \omega+w \sin (\omega \tau) & =0,
\end{aligned}
$$

which leads to [46]

$$
\begin{aligned}
w_{\mathrm{H}}(\omega) & =\frac{\left(\omega^{2}-1\right)^{2}+4 \zeta^{2} \omega^{2}}{2\left(\omega^{2}-1\right)}, \\
\tau_{\mathrm{H}}(\omega, j) & =\frac{2}{\omega}\left(j \pi-\arctan \left(\frac{\omega^{2}-1}{2 \zeta \omega}\right)\right), \\
\Omega_{\mathrm{H}}(\omega, j) & =\frac{2 \pi}{\tau_{\mathrm{H}}(\omega, j)} .
\end{aligned}
$$

Equation (17) defines a family of curves called stability lobes with lobe number $j \in \mathbb{N}$. The curves are parameterized by $\omega \in(0, \infty)$. The stability lobes are conventionally depicted in the plane of the technological parameters such as the (dimensionless) angular velocity $\Omega$ and the (dimensionless) chip width $w$, which results in so-called stability lobe diagrams (or stability charts) as shown in Fig. 3(a) for $\zeta=0.02$. Gray shading indicates the linearly stable region $0<w<w_{\mathrm{H}}$ that is associated with chatter-free cutting process. The stability charts help manufacturing engineers in the selection of optimal technological parameters where machine tool chatter is avoided and the material removal rate (proportional to $\Omega w$ ) is high.

Along the linear stability boundaries (17), Hopf bifurcation occurs (as indicated by subscript $\mathrm{H}$ ). The theory of Hopf bifurcation in delay-differential equations is covered by [21, 22, 23, $20,24]$, while its analysis for turning processes can be found in $[11,12,13,14]$. According to [14], the Hopf bifurcation is subcritical for the power law (5) and for the cubic cutting force characteristics (6) with realistic parameters. The subcriticality implies that the Hopf bifurcation gives rise to an unstable periodic orbit (unstable limit cycle) in the vicinity of the linearly stable equilibrium. The approximate dimensionless angular frequency of the limit cycle is $\omega \in(0, \infty)$.

This phenomenon is illustrated qualitatively in Fig. 3(b) where the phase portrait of Eq. (13) is depicted. Although the phase space of Eq. (13) is infinite-dimensional, the trajectories can still be illustrated using three coordinates: $x, x^{\prime}$ and $x_{\infty}$, where $x_{\infty}$ refers to the remaining infinite dimensions. According to Fig. 3(b), the basin of attraction of the linearly stable equilibrium does not cover the whole phase space when the unstable limit cycle exists, thus the equilibrium is not stable in the global sense. Depending on initial conditions and perturbations (such as material inhomogeneity or external excitation), the system may leave the basin of attraction and converge to another stable solution. This solution is large-amplitude chatter 

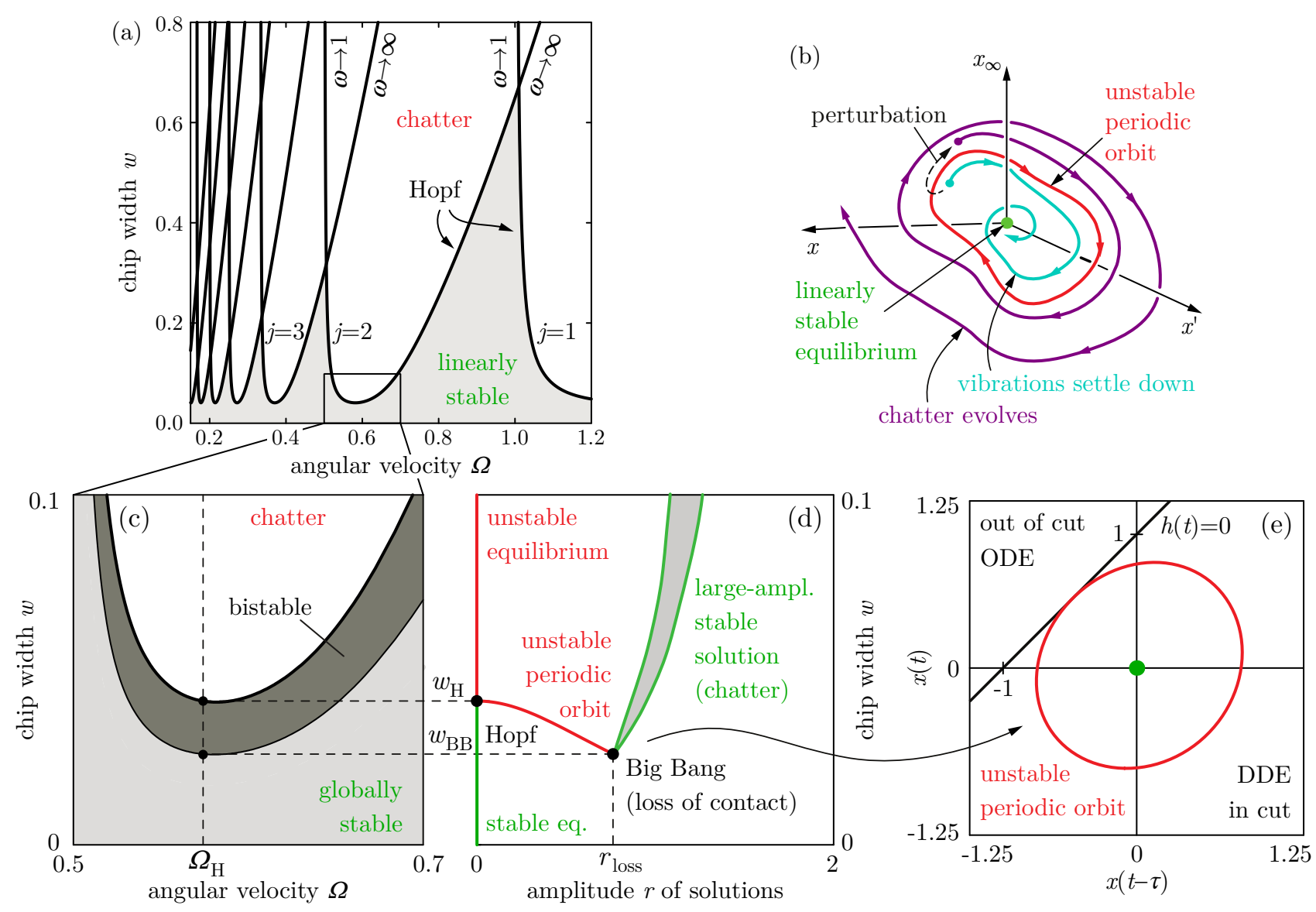

Figure 3: (a) Stability lobe diagram of turning; (b) illustration of the trajectories near the unstable periodic orbit arising from Hopf bifurcation; (c) illustration of the bistable region; (d) the corresponding bifurcation scenario; (e) the periodic orbit at loss of contact.

- an intermittent motion where the tool leaves and gets back into the workpiece repeatedly [11, 12, 13, 47, 14, 48].

The parameter domain where stable stationary cutting and large-amplitude chatter coexist due to the existence of an unstable periodic orbit is referred to as region of bistability (or unsafe zone). It is important to emphasize that the phenomenon of bistability is caused by the nonlinearity in the system. If a linear cutting force characteristics (i.e. $\eta_{m}=0$ for $m \geq 2$ ) was considered in Eq. (13), then the bistable region would disappear. However, the existence of the bistable region was verified experimentally in $[12,49,16,43]$, while its numerical analysis for milling was published in [19]. From practical point of view, bistability should be avoided, since chatter may occur despite linear stability if the cutting process is perturbed as illustrated in Fig. 3(b). The bistable region is shown qualitatively in Fig. 3(c) with dark grey shading. Light grey shading indicates the globally stable region, where the cutting process is safe and no machine tool vibrations evolve.

The corresponding bifurcation scenario is discussed in [48] in detail and is illustrated in Fig. 3(d). The bifurcation parameter is chosen to be the dimensionless chip width $w$, while the dimensionless angular velocity $\Omega=\Omega_{\mathrm{H}}$ is fixed (see the dashed line in Fig. 3(c)). As shown by Fig. 3(d), the amplitude $r$ of the unstable limit cycle born from Hopf bifurcation increases with decreasing bifurcation parameter. At the critical amplitude $r_{\text {loss }}$, the (unstable) oscillations get so large that the tool loses contact with the workpiece as illustrated in Fig. 1(b). In such cases, the chip thickness drops to zero and to negative values $(h(t) \leq 0)$ that is associated with nonsmoothness in the cutting force characteristics (2) and with nonsmooth dynamics: the governing equation switches from a delay-differential equation to an ordinary differential equation. The unstable periodic orbit corresponding to $r_{\text {loss }}$ is illustrated in Fig. 3(e).

According to [48], the unstable periodic orbit undergoes a special nonsmooth fold bifurcation (called Big Bang bifurcation) at $r_{\text {loss }}$. The nonsmooth fold gives rise to a large-amplitude stable solution that corresponds to machine tool chatter, which may be a periodic or chaotic motion that is stable in the dynamical sense [48]. Thus, the linearly stable equilibrium coexists with the large-amplitude stable solution between the Hopf bifurcation point $w=w_{\mathrm{H}}$ and the Big Bang bifurcation point $w=w_{\mathrm{BB}}$, see Fig. 3(d). The bistable region is $w_{\mathrm{H}}<w<w_{\mathrm{BB}}$, and its boundary $w=w_{\mathrm{BB}}$ can be obtained by locating the point where the unstable periodic solution first satisfies the condition $h(t)=0$ associated with loss of contact, cf. Fig. 3(e).

The rest of the paper deals with the computation of the unstable limit cycle and the bistable region. In [11, 12, 13, 14], approximate analytical formulas were given for the bistable re- 
gion. These analyses used a cubic (expansion of the) cutting force characteristics and were valid in the vicinity of Hopf bifurcation only. Thus, the formulas for the bistable region are inaccurate in some cases (for large bistable regions or in the presence of higher-order nonlinearities). The rest of the paper is devoted to extending these results by the derivation of more accurate analytical formulas for the bistable region while considering higher-order nonlinearities. A similar effort can be found in [16] for the power law (5) - now we consider a general cutting force characteristics.

\section{Method of Averaging}

In order to compute the bistable region, the amplitude of the unstable limit cycle must be determined. In what follows, we use the Krylov-Bogoliubov-Mitropolsky method of averaging [33] to find the amplitude. The key point of the analysis is the approximation of the limit cycle by its first harmonic. At Hopf bifurcation $\left(w=w_{\mathrm{H}}\right)$, the linear part of Eq. (13) indeed has harmonic solution. The linear part reads

$$
x^{\prime \prime}(t)+2 \zeta x^{\prime}(t)+x(t)=w_{\mathrm{H}}(x(t-\tau)-x(t)),
$$

whereas its solution can be given in the form

$$
\begin{gathered}
x(t)=r \cos (\omega t+\varphi), \\
x^{\prime}(t)=-r \omega \sin (\omega t+\varphi),
\end{gathered}
$$

where the amplitude $r$ and the phase $\varphi$ depend on initial conditions. The solution of the nonlinear system (13) is sought for in the same form as in Eq. (19), but with time-dependent amplitude $\hat{r}(t)$ and phase $\hat{\varphi}(t)$ :

$$
\begin{aligned}
x(t) & =\hat{r}(t) \cos (\omega t+\hat{\varphi}(t)), \\
x^{\prime}(t) & =-\hat{r}(t) \omega \sin (\omega t+\hat{\varphi}(t)) .
\end{aligned}
$$

The constant approximation of $\hat{r}(t)$ and $\hat{\varphi}(t)$ implies the harmonic approximation of the limit cycle. Thus first, we transform Eq. (13) and express it in terms of $\hat{r}(t)$ and $\hat{\varphi}(t)$. Then, we search for constant (approximate) solutions.

By multiplying the left-hand side of the expressions in Eq. (20) by $\cos (\omega t+\hat{\varphi}(t))$ and $-\sin (\omega t+\hat{\varphi}(t)) / \omega$, respectively, and by adding them, we get the amplitude as

$$
\hat{r}(t)=x(t) \cos (\omega t+\hat{\varphi}(t))-x^{\prime}(t) \frac{1}{\omega} \sin (\omega t+\hat{\varphi}(t)),
$$

while the following identity can also be obtained in a similar manner:

$$
0 \equiv x(t) \sin (\omega t+\hat{\varphi}(t))+x^{\prime}(t) \frac{1}{\omega} \cos (\omega t+\hat{\varphi}(t)) .
$$

Differentiation of Eq. (21) with respect to time and using Eqs. (13) and (22) yields the Lie derivative of the amplitude $\hat{r}(t)$ in the form

$$
\begin{aligned}
\hat{r}^{\prime}(t)=- & \frac{1}{\omega}\left(\omega^{2} x(t)-2 \zeta x^{\prime}(t)-x(t)\right. \\
& \left.+w \sum_{m=1}^{\infty} \eta_{m}(x(t-\tau)-x(t))^{m}\right) \sin (\omega t+\hat{\varphi}(t)) .
\end{aligned}
$$

Similarly, the Lie derivative of the phase $\hat{\varphi}(t)$ can be expressed via differentiation of Eq. (22) and using Eqs. (13) and (21), which gives

$$
\begin{aligned}
\hat{\varphi}^{\prime}(t)= & \frac{1}{\omega \hat{r}(t)}\left(\omega^{2} x(t)-2 \zeta x^{\prime}(t)-x(t)\right. \\
& \left.+w \sum_{m=1}^{\infty} \eta_{m}(x(t-\tau)-x(t))^{m}\right) \cos (\omega t+\hat{\varphi}(t)) .
\end{aligned}
$$

From this point on, Eq. (23) is considered only to calculate the bistable region from the amplitude of the limit cycle.

Now, we make the main assumption of the subsequent analysis. We suppose that the amplitude $\hat{r}(t)$ and the phase $\hat{\varphi}(t)$ vary slowly in time (which is reasonable as we seek for their constant approximation). Note that this can also be considered as a multiple scales assumption. The slow variation implies $\hat{r}(t-\tau) \approx \hat{r}(t)$ and $\hat{\varphi}(t-\tau) \approx \hat{\varphi}(t)$. Then, the chip thickness variation $x(t-\tau)-x(t)$ in Eq. (23) can be approximated using Eq. (20) as

$$
x(t-\tau)-x(t) \approx \hat{r}(t) \sqrt{4 \sin ^{2}\left(\frac{\omega \tau}{2}\right)} \cos (\omega t+\hat{\varphi}(t)+\psi),
$$

where $\psi$ is a phase shift satisfying

$$
\sin \psi=-\frac{\sin (\omega \tau)}{\sqrt{4 \sin ^{2}\left(\frac{\omega \tau}{2}\right)}}
$$

Substituting Eqs. (20) and (25) into Eq. (23) leads to the differential equation for $\hat{r}(t)$ in the form

$$
\begin{aligned}
& \hat{r}^{\prime}(t)=-2 \zeta \hat{r}(t) \sin ^{2}(\omega t+\hat{\varphi}(t)) \\
&+\left(\frac{1}{\omega}-\omega\right) \hat{r}(t) \cos (\omega t+\hat{\varphi}(t)) \sin (\omega t+\hat{\varphi}(t)) \\
& \quad-\frac{1}{\omega} w \sum_{m=1}^{\infty} \eta_{m} r^{m}(t)\left(4 \sin ^{2}\left(\frac{\omega \tau}{2}\right)\right)^{m / 2} \\
& \quad \times \cos ^{m}(\omega t+\hat{\varphi}(t)+\psi) \sin (\omega t+\hat{\varphi}(t)) .
\end{aligned}
$$

Notice that Eq. (27) is a nonautonomous ordinary differential equation that does not involve time delay anymore.

Now, the method of averaging is applied to Eq. (27). The theoretical background and examples for this method can be found in $[33,34,35,36,37,38,39,40,41]$. Equation (27) is averaged by integrating both sides from 0 to $2 \pi / \omega$ with respect to $t$ while considering $\hat{r}$ and $\hat{\varphi}$ time-independent. Multiplying the resulting equation by $\omega /(2 \pi)$, the averaged system is obtained in the form

$$
\bar{r}^{\prime}(t)=-\zeta \bar{r}(t)-\frac{w}{2 \pi \omega} \sum_{m=1}^{\infty} \eta_{m} \bar{r}^{m}(t)\left(4 \sin ^{2}\left(\frac{\omega \tau}{2}\right)\right)^{m / 2} \beta_{m},
$$

where $\bar{r}$ is the averaged amplitude and $\beta_{m}$ is defined by

$$
\beta_{m}=\int_{0}^{2 \pi / \omega} \cos ^{m}(\omega t+\psi) \sin (\omega t) \omega \mathrm{d} t, \quad m \in \mathbb{Z}^{+} .
$$


Note that the phase $\hat{\varphi}$ is omitted in $\beta_{m}$, since a phase shift does not modify the integral of a periodic function over its time period. The expression of $\beta_{m}$ can be simplified to

$$
\beta_{2 k-1}=-\frac{\pi \sin \psi}{4^{k-1}}\left(\begin{array}{c}
2 k-1 \\
k
\end{array}\right), \quad \beta_{2 k}=0, \quad k \in \mathbb{Z}^{+} .
$$

\section{Amplitude of the Limit Cycle}

Consider the constant approximation $\hat{r}(t) \approx \bar{r}(t) \approx r$ of the amplitude that corresponds to the harmonic approximation of the limit cycle. Substitution of $\bar{r}(t) \equiv r$ and Eq. (30) into Eq. (28) gives

$$
\begin{aligned}
-\zeta r+\frac{w}{2 \pi \omega} & \sum_{k=1}^{\infty} \eta_{2 k-1} r^{2 k-1} \\
& \times\left(4 \sin ^{2}\left(\frac{\omega \tau}{2}\right)\right)^{(2 k-1) / 2} \frac{\pi \sin \psi}{4^{k-1}}\left(\begin{array}{c}
2 k-1 \\
k
\end{array}\right)=0 .
\end{aligned}
$$

Now, we substitute $\zeta$ from Eq. (16), we use Eq. (26) and divide by $r \sin (\omega \tau) /(2 \omega) \neq 0$. These yield

$$
w_{\mathrm{H}}-w \sum_{k=1}^{\infty}\left(\begin{array}{c}
2 k-1 \\
k
\end{array}\right) \eta_{2 k-1}\left(r^{2} \sin ^{2}\left(\frac{\omega \tau}{2}\right)\right)^{k-1}=0 .
$$

The calculation of the approximate amplitude $r$ of the periodic orbit is reduced to finding a positive real root of the polynomial in Eq. (32). If the nonlinearity in Eq. (13) is a polynomial of low order, then Eq. (32) can be solved analytically for $r^{2} \sin ^{2}(\omega \tau / 2)$ to obtain $r$. The cubic cutting force characteristics (6), that is, $\eta_{m}=0$ for $m \geq 4$ leads to

$$
r_{3 \mathrm{rd}}(w)=\sqrt{-\frac{w-w_{\mathrm{H}}}{3 \eta_{3} \sin ^{2}\left(\frac{\omega \tau}{2}\right) w}} .
$$

For quintic nonlinearity ( $\eta_{m}=0$ for $m \geq 6$ ), Eq. (32) gives

$$
r_{5 \mathrm{th}}(w)=\sqrt{\frac{-3 \eta_{3} w+\sqrt{9 \eta_{3}^{2} w^{2}-40 \eta_{5} w\left(w-w_{\mathrm{H}}\right)}}{20 \eta_{5} \sin ^{2}\left(\frac{\omega \tau}{2}\right) w}} .
$$

In a similar manner, Eq. (32) can be solved analytically by computer algebra for 7 th, 9 th and 11 th-order nonlinearities as well, but the resulting formulas are too long to be listed here. Above 11th order and for complete Taylor series, Eq. (32) can be solved numerically for the amplitude.

The amplitude $r$ of the limit cycle as a function of the dimensionless chip width $w$ is shown in Fig. 4(b). Here, the damping ratio is $\zeta=0.02$ and the bifurcation diagrams are computed for $\Omega=0.77486$ (i.e., $j=2, \omega=1.1903$ ) as indicated by the dashed line in the corresponding stability chart in Fig. 4(a). The exponential cutting force characteristics (5) is considered with feed per revolution $h_{0}=0.05 \mathrm{~mm}$. The cutting coefficients are $b_{1}=176 \mathrm{~N} / \mathrm{mm}^{2}, b_{2}=4386 \mathrm{~N} / \mathrm{mm}^{3}, b_{3}=-1291 / \mathrm{mm}$, $b_{4}=0$, which were identified in [50] for milling aluminum with four cutting teeth. In order to show the effect of higher-order nonlinear terms, the nonlinearity in Eq. (13) is truncated after the $3 \mathrm{rd}, 5 \mathrm{th}, 7 \mathrm{th}, 9$ th and 11 th-order terms as shown by purple, blue, green, orange and red colors, respectively. The analytical bifurcation diagrams obtained from Eq. (32) are indicated by solid lines. The amplitude corresponding to the full nonlinear term in Eq. (13) is shown by black color. This black curve was obtained by simplifying the sum in Eq. (32) to a closed-form expression via computer algebra and by creating a contour plot based on Eq. (32) afterwards.

The analytical results were verified by numerical continuation using DDE-BIFTool [51]. The numerical bifurcation diagrams showing half of the peak-to-peak amplitude of the limit cycle are indicated by dashed lines with the same color scheme as that of their analytical counterparts. The analytical and numerical results agree well, the solid and dashed branches overlap. This justifies that the periodic solution of Eq. (13) is indeed nearly harmonic.

\section{Region of Bistability}

Expressions for the amplitude of the limit cycle allow the calculation of the bistable region. Figure 4(b) shows that higherorder nonlinearities have significant effect on the amplitude and hence on the size of the bistable region. Thus, we extend the results of [14] and derive formulas for the size of the bistable region by taking into account the full nonlinear term in Eq. (13).

According to [48], the boundary $w=w_{\mathrm{BB}}$ of the bistable region is located where the amplitude of the unstable periodic oscillations gets so large that the flyover shown in Fig. 1(b) takes place. This happens when the chip thickness drops to zero. The chip thickness $h(t)$ can be expressed using Eqs. (8), (10) and (25). Then, substitution of $\hat{r}(t) \approx r$ and $\cos (\omega t+\hat{\varphi}(t)+\psi)=-1$ into $h(t)=0$ gives the smallest amplitude $r_{\text {loss }}$ where loss of contact takes place in the form

$$
r_{\mathrm{loss}}=\frac{1}{\sqrt{4 \sin ^{2}\left(\frac{\omega \tau}{2}\right)}}
$$

Note that the bifurcation diagrams in Fig. 4(b) are valid for $0 \leq r \leq r_{\text {loss }}$ only (where $r_{\text {loss }}=0.503$ in this example).

When the chip width is decreased to $w=w_{\mathrm{BB}}$, the amplitude of the unstable limit cycle becomes $r_{\text {loss. }}$. Thus, loss of contact takes place and the boundary of the bistable region is reached. After substitution of $w=w_{\mathrm{BB}}, r=r_{\text {loss }}$ and Eq. (35) into Eq. (32), the boundary of the bistable region becomes

$$
w_{\mathrm{BB}}=w_{\mathrm{H}}\left(\sum_{k=1}^{\infty} \frac{1}{4^{k-1}}\left(\begin{array}{c}
2 k-1 \\
k
\end{array}\right) \eta_{2 k-1}\right)^{-1}
$$

The size of the bistable region can be compared to the size of the linearly stable region by introducing the ratio

$$
R_{\mathrm{bist}}=\frac{w_{\mathrm{H}}-w_{\mathrm{BB}}}{w_{\mathrm{H}}}
$$



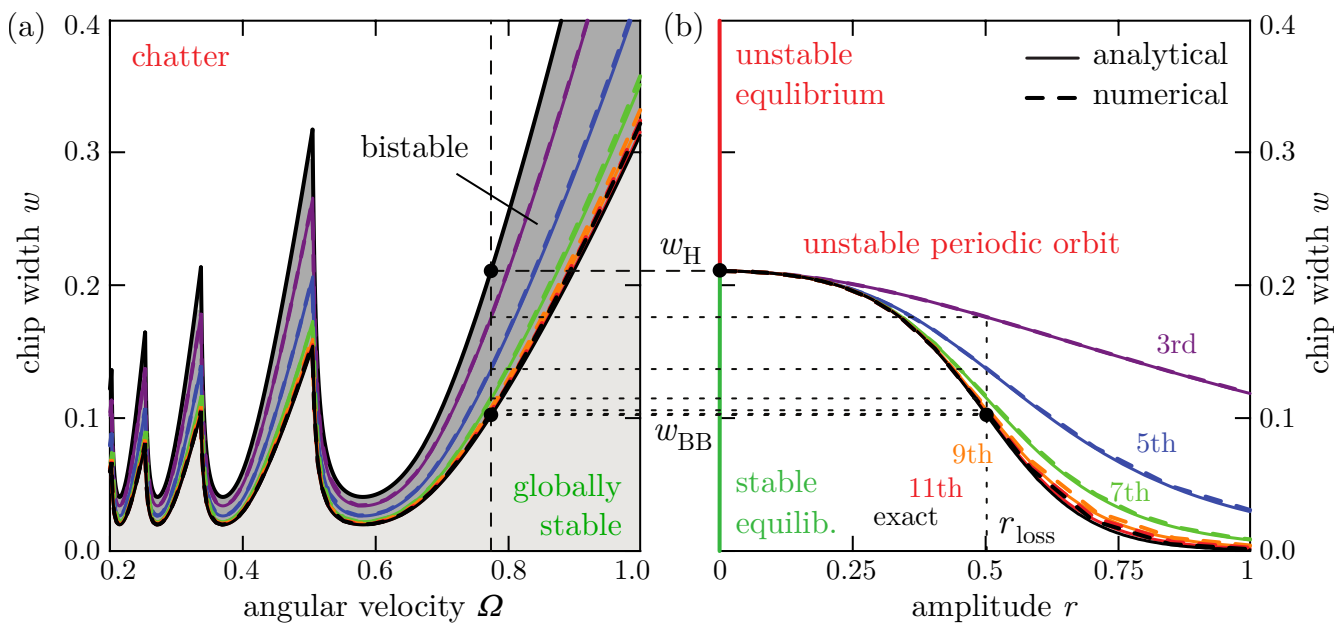

Figure 4: (a) Stability chart of the turning model (13); (b) bifurcation diagrams for different orders of nonlinearity. Solid lines indicate analytical results obtained from Eq. (32), dashed lines show the results of numerical continuation by DDE-BIFTooL.

that can be expressed as

$$
\begin{aligned}
R_{\text {bist }} & =1-\left(\sum_{k=1}^{\infty} \frac{1}{4^{k-1}}\left(\begin{array}{c}
2 k-1 \\
k
\end{array}\right) \eta_{2 k-1}\right)^{-1} \\
& =1-\left(1+\frac{3}{4} \eta_{3}+\frac{10}{16} \eta_{5}+\frac{35}{64} \eta_{7}+\frac{126}{256} \eta_{9}+\ldots\right)^{-1} .
\end{aligned}
$$

Note that the approximate size $R_{\text {bist }}$ of the bistable region is independent of the location of the Hopf bifurcation point (i.e., independent of $\omega$ or $\Omega$ ). Besides, recall that the bistable region disappears for a linear cutting force characteristics. Accordingly, Eq. (38) shows that $R_{\text {bist }}=0$ if $\eta_{\mathrm{m}}=0$ for $m \geq 2$.

For the power law cutting force characteristics (5), substitution of Eq. (15) into Eq. (38) and simplification give

$$
R_{\mathrm{bist}}=1-\frac{\sqrt{\pi} \Gamma(v+2)}{2^{v+1} \Gamma\left(v+\frac{1}{2}\right)},
$$

where $\Gamma$ denotes the Euler gamma function. For the cubic cutting force characteristics (6), the resulting expression is

$$
R_{\text {bist }}=\frac{3 \rho_{3} h_{0}^{2}}{4 \rho_{1}+8 \rho_{2} h_{0}+15 \rho_{3} h_{0}^{2}} .
$$

In the case of the exponential cutting force characteristics (7), the size of the bistable region becomes

$$
R_{\text {bist }}=1-\frac{b_{1} h_{0}+b_{2} h_{0} \mathrm{e}^{b_{3} h_{0}}}{b_{1} h_{0}+2 \frac{b_{2}}{b_{3}} \mathrm{e}^{b_{3} h_{0}} I_{1}\left(b_{3} h_{0}\right)},
$$

where $I_{1}$ is the modified Bessel function of the first kind.

Using formulas (38)-(41), the bistable region can be computed analytically as shown by the solid lines in Fig. 4(a). Here, the same color scheme is used as in Fig. 4(b), and the analytical results were again verified by numerical continuation with DDE-BIFTool, see the overlapping dashed lines. In what follows, we evaluate formulas (39)-(41) and compare them to existing results in the literature.

\section{Results and Discussion}

It must be mentioned that formula (39) associated with the power law cutting force characteristics (5) was already derived by Tamás Kalmár-Nagy in [16] and also by Pankaj Wahi according to personal communications [52]. In their works an equivalent approach, the method of harmonic balance was used. Equation (39) shows that, for the power law, the size of the bistable region depends on the cutting exponent $v$ only. Assuming $v=3 / 4$ (i.e., the well-known three-quarter rule), the bistable region occupies $6.5 \%$ of the linearly stable region. That is, the bistable region is small independently of any technological parameters. For other cutting force characteristics (that were not discussed in [16]), the bistable region is more significant and an accurate closed-form formula for its prediction becomes more relevant. Formula (38) introduced in this paper is valid for any cutting force characteristics.

For the cubic polynomial (6) and the exponential cutting force characteristics (7), the bistable region depends on the feed $h_{0}$ per revolution according to Eqs. (40) and (41). Thus, the bistable region may be significantly larger than $6.5 \%$ of the linearly stable region, which was also confirmed by experiments with large feed rates in [43]. The size of the bistable region is plotted against the feed $h_{0}$ per revolution in Fig. 5 for the power law (green), the cubic polynomial (blue), and the exponential characteristics (red). The cutting force parameters are $v=3 / 4$ for the power law, $\rho_{1}=6.1096 \times 10^{3} \mathrm{~N} / \mathrm{mm}^{2}$, $\rho_{2}=-5.41416 \times 10^{4} \mathrm{~N} / \mathrm{mm}^{3}, \rho_{3}=2.03769 \times 10^{5} \mathrm{~N} / \mathrm{mm}^{4}$ for the cubic polynomial (that were identified experimentally in [15] for a milling tool of four teeth), and $b_{1}=176 \mathrm{~N} / \mathrm{mm}^{2}$, $b_{2}=4386 \mathrm{~N} / \mathrm{mm}^{3}, b_{3}=-1291 / \mathrm{mm}, b_{4}=0$ for the exponential function (the same as in Fig. 4). For the cubic and the exponential cutting force expressions, the bistable region becomes significant: it occupies about $50 \%$ of the linearly stable region at certain critical feed per revolutions.

This justifies the relevance of considering other cutting force characteristics than the power law. To the best knowledge of the 


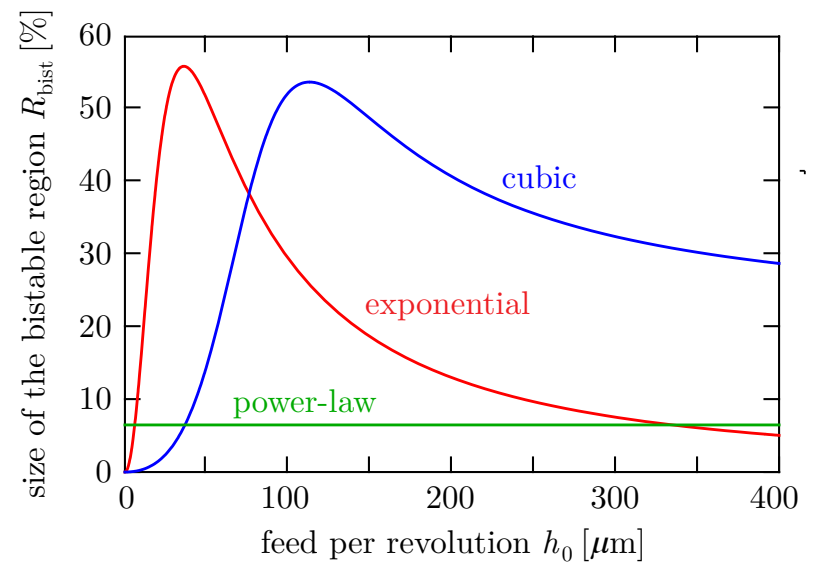

Figure 5: The size of the bistable region as a function of the feed per revolution for various cutting force characteristics.

authors, the exponential cutting force expression has not been analyzed previously from nonlinear dynamics point of view. On the other hand, the cubic characteristics has been studied extensively in the literature. Cubic nonlinearities and bistability in orthogonal cutting were first discussed in [15] via the method of harmonic balance without deriving closed-form formulas for the bistable region. Later, it was analyzed by center manifold reduction in $[11,12,13,14]$ and by the method of multiple scales in [30, 31, 32]. In [14], it was shown that the size of the bistable region occupies approximately $3 / 4 \eta_{3}$ portion of the linearly stable region. These analyses, however, are valid in the vicinity of the Hopf bifurcation only. Farther away, the results become inaccurate and cannot predict the size of a large bistable region accurately (an overestimation by a factor of 2 was reported in [14] at critical feed per revolutions).

In [53], a higher-order estimation for the amplitude of limit cycles was introduced, which is still valid farther from the Hopf bifurcation. This way, the results of $[11,14]$ could be refined and larger bistable regions could also be predicted accurately. This method is based on center manifold reduction and uses the property $\lim _{w \rightarrow 0} r=\infty$ of the limit cycle (that is also captured by Eqs. (32), (33) and (34)). This approach gives $3 / 4 \eta_{3} /\left(1+3 / 4 \eta_{3}\right)$ for the size of the bistable region, which is the same that obtained by averaging, see the truncation of Eq. (38) for cubic nonlinearity.

The method of averaging is not restricted to the cubic approximation of the cutting force characteristics but can be applied to higher-order nonlinearities as well. It should be mentioned, however, that the coefficients of higher-order terms are more uncertain and harder-to-measure from engineering point of view. In the meantime, the experimentally determined size of the bistable zone can be used well for the identification of these higher-order terms by applying the results in an inverse way as shown in [43]. Consideration of higher-order terms is theoretically possible by center manifold reduction and by the method of multiple scales, too, but the calculations become unmanageably complicated.

Note that the approximate solution (20) obtained by the method of averaging has a certain error and timescale of validity. In order to investigate this error and timescale, let us rewrite Eq. (13) in the form

$$
\begin{aligned}
& x^{\prime \prime}(t)+2 \zeta x^{\prime}(t)+x(t)-w_{\mathrm{H}}(x(t-\tau)-x(t)) \\
= & \varepsilon\left(x(t-\tau)-x(t)+\frac{w}{w-w_{\mathrm{H}}} \sum_{m=2}^{\infty} \eta_{m}(x(t-\tau)-x(t))^{m}\right)
\end{aligned}
$$

with $\varepsilon=w-w_{\mathrm{H}}$. This form satisfies that for $\varepsilon=0 \mathrm{Eq}$. (42) has the harmonic solution of the form (19). Thus, form (42) reveals that, for $\varepsilon \neq 0$, solution (20) has a timescale of validity $1 / \varepsilon$ and is accurate with error $O(\varepsilon)$ [40]. Here, the timescale of validity is not of importance, because it is associated with the accuracy of the phase $\hat{\varphi}(t)$. Since the amplitude of the unstable limit cycle was required only to obtain the bistable region, the phase was not even determined and its accuracy is irrelevant from practical point of view. As for the amplitude, its error turned out to be practically negligible, see Fig. 4.

Apart from the method of averaging, the amplitude (32) of the limit cycle and the bistable region can also be derived using Melnikov's integral. This method serves equivalent results to averaging, which is shown in the Appendix for the sake of completeness. The method of averaging and Melnikov's integral serve rigorous results with the possibility of giving error estimates for the obtained approximate solutions.

In summary, the method of averaging provides simple closed-form formulas for the size of the bistable region in metal cutting even in the presence of higher-order nonlinearities. Numerical continuation confirmed the accuracy of the analytical results even at critical feed per revolutions where the bistable region is large. Although the key point of the analysis is the harmonic approximation of the limit cycle, the nonlinearity was expanded into Taylor series as in center manifold reduction. However, it is not necessary to truncate the series after finitely many terms: it is possible to obtain the size of the bistable region analytically for a complete Taylor series as well, see Eqs. (39) and (41). The amplitude of the limit cycle can be determined analytically for up to 11th-order nonlinearity and numerically for any order using Eq. (32). Whereas the result (38) for the size of the bistable region is analytical for any order of nonlinearity, and this result is relevant from practical point of view.

\section{Acknowledgements}

This work has been supported by the ÚNKP-16-3-I. New National Excellence Program of the Ministry of Human Capacities. The research leading to these results has received funding from the European Research Council under the European Union's Seventh Framework Programme (FP/20072013) / ERC Advanced Grant Agreement n. 340889.

\section{Appendix A. Solution by Melnikov's Integral}

Finally, we show that Eq. (32) for the approximate amplitude of the limit cycle can also be obtained using Melnikov's integral. This method is discussed in [42] in detail, and its core idea 
is decomposing the dynamical system to a Hamiltonian system and a perturbation. The unperturbed Hamiltonian system is of form

$$
\begin{aligned}
& x_{1}^{\prime}(t)=\frac{\partial H}{\partial x_{2}}(t), \\
& x_{2}^{\prime}(t)=-\frac{\partial H}{\partial x_{1}}(t),
\end{aligned}
$$

where the Hamiltonian $H$ is constant along the trajectories of the system, since its Lie derivative is zero: $H^{\prime}(t)=\frac{\partial H}{\partial x_{1}}(t) x_{1}^{\prime}(t)+\frac{\partial H}{\partial x_{2}}(t) x_{2}^{\prime}(t) \equiv 0$. For Eq. (13), the unperturbed Hamiltonian system is given by Eq. (18), thus with $x_{1}(t)=x(t)$ and $x_{2}(t)=x^{\prime}(t)$ the Hamiltonian becomes

$$
H(t)=\frac{x_{1}^{2}(t) \omega^{2}}{2}+\frac{x_{2}^{2}(t)}{2} .
$$

The solution of the unperturbed system is given by Eq. (19) and the corresponding trajectories are closed curves in the plane $\left(x_{1}, x_{2}\right)$. According to [42], one of these closed trajectories is preserved as a limit cycle in the perturbed nonlinear system, which, in some sense, conserves the property that $H$ is constant along the closed trajectory.

The perturbed system can be given in the form

$$
\begin{aligned}
& x_{1}^{\prime}(t)=\frac{\partial H}{\partial x_{2}}(t)+g_{1}(t), \\
& x_{2}^{\prime}(t)=-\frac{\partial H}{\partial x_{1}}(t)+g_{2}(t),
\end{aligned}
$$

where the perturbation is

$$
\begin{aligned}
& g_{1}(t)=0, \\
& g_{2}(t)=\left(w-w_{\mathrm{H}}\right) \Delta x_{1}(t)+w \sum_{m=2}^{\infty} \eta_{m} \Delta x_{1}^{m}(t)
\end{aligned}
$$

with $\Delta x_{1}(t)=x_{1}(t-\tau)-x_{1}(t)$, see Eq. (13). In this perturbed system, the Lie derivative of the associated Hamiltonian is $H^{\prime}(t)=\frac{\partial H}{\partial x_{1}}(t) x_{1}^{\prime}(t)+\frac{\partial H}{\partial x_{2}}(t) x_{2}^{\prime}(t)=g_{2}(t) x_{1}^{\prime}(t)-g_{1}(t) x_{2}^{\prime}(t)$. According to [42], the condition for a closed trajectory of the unperturbed system (that satisfies $H(t)=$ const) to be preserved in the perturbed system is given by Melnikov's integral:

$$
\oint_{L}\left(g_{2}(t) x_{1}^{\prime}(t)-g_{1}(t) x_{2}^{\prime}(t)\right) \mathrm{d} t=0,
$$

where $L$ denotes the closed trajectory of the perturbed system (i.e., the limit cycle).

Substitution of Eq. (A.4) yields

$$
\oint_{L}\left(\left(w-w_{\mathrm{H}}\right) \Delta x_{1}(t)+w \sum_{m=2}^{\infty} \eta_{m} \Delta x_{1}^{m}(t)\right) x_{1}^{\prime}(t) \mathrm{d} t=0 \text {. }
$$

Now, we approximate the limit cycle $L$ by the corresponding trajectory of the unperturbed system: we substitute the harmonic solution (19) into Eq. (A.6) and carry out the integration over $[0,2 \pi / \omega]$. We obtain

$$
\begin{aligned}
-\left(w-w_{\mathrm{H}}\right) r^{2} & \sqrt{4 \sin ^{2}\left(\frac{\omega \tau}{2}\right)} \beta_{1} \\
& -w r \sum_{m=2}^{\infty} \eta_{m} r^{m}\left(4 \sin ^{2}\left(\frac{\omega \tau}{2}\right)\right)^{m / 2} \beta_{m}=0,
\end{aligned}
$$

where $\beta_{m}$ is defined by Eq. (29). Substituting the simplified expression (30) of $\beta_{m}$, Eq. (A.7) becomes equivalent to Eq. (32) obtained by the method of averaging. Consequently, Eq. (32) and all the subsequent equations can alternatively be derived using Melnikov's integral, while Figs. 4 and 5 can also be obtained by this method.

\section{References}

[1] S. A. Tobias, W. Fishwick, Theory of regenerative machine tool chatter, The Engineer (1958) 199-203, 238-239.

[2] J. Tlusty, M. Polacek, The stability of the machine tool against selfexcited vibration in machining, in: ASME Production Engineering Research Conference, Pittsburgh, PA, USA, 1963, pp. 454-465.

[3] M. Wiercigroch, E. Budak, Sources of nonlinearities, chatter generation and suppression in metal cutting, Philosophical Transactions of the Royal Society A: Mathematical, Physical and Engineering Sciences 359 (1781) (2001) 663-693.

[4] N. D. Sims, B. Mann, S. Huyanana, Analytical prediction of chatter stability for variable pitch and variable helix milling tools, Journal of Sound and Vibration 317 (3-5) (2008) 664-686.

[5] P. Wahi, A. Chatterjee, Self-interrupted regenerative metal cutting in turning, International Journal of Non-Linear Mechanics 43 (2) (2008) 111123.

[6] E. A. Butcher, O. Bobrenkov, E. Bueler, P. Nindujarla, Analysis of milling stability by the Chebyshev Collocation Method: algorithm and optimal stable immersion levels, Journal of Computational and Nonlinear Dynamics 4 (3) (2009) 031003 (12 pages).

[7] Y. Altintas, Manufacturing Automation - Metal Cutting Mechanics, Machine Tool Vibrations and CNC Design, Second Edition, Cambridge University Press, Cambridge, 2012.

[8] X. Liu, N. Vlajic, X. Long, G. Meng, B. Balachandran, State-dependent delay influenced drill-string oscillations and stability analysis, Journal of Vibration and Acoustics 136 (5) (2014) 051008 (9 pages).

[9] G. Totis, P. Albertelli, M. Sortino, M. Monno, Efficient evaluation of process stability in milling with Spindle Speed Variation by using the Chebyshev Collocation Method, Journal of Sound and Vibration 333 (3) (2014) 646-668.

[10] Y. Yan, J. Xu, M. Wiercigroch, Regenerative chatter in a plunge grinding process with workpiece imbalance, International Journal of Advanced Manufacturing Technology 89 (9-12) (2016) 2845-2862.

[11] G. Stépán, T. Kalmár-Nagy, Nonlinear regenerative machine tool vibrations, in: Proceedings of DETC'97, ASME Design and Technical Conferences, Sacramento, CA, USA, 1997, pp. 1-11.

[12] T. Kalmár-Nagy, J. R. Pratt, M. A. Davies, M. D. Kennedy, Experimental and analytical investigation of the subcritical instability in turning, in: Proceedings of the DETC'99 17th ASME Biennial Conference on Mechanical Vibration and Noise, no. DETC99/VIB-8060, Las Vegas, NA, USA, 1999

[13] T. Kalmár-Nagy, G. Stépán, F. C. Moon, Subcritical Hopf bifurcation in the delay equation model for machine tool vibrations, Nonlinear Dynamics 26 (2001) 121-142.

[14] Z. Dombóvári, R. E. Wilson, G. Stépán, Estimates of the bistable region in metal cutting, Proceedings of the Royal Society A - Mathematical, Physical and Engineering Sciences 464 (2008) 3255-3271.

[15] H. M. Shi, S. A. Tobias, Theory of finite amplitude machine tool instability, International Journal of Machine Tool Design and Research 24 (1) (1984) 45-69.

[16] T. Kalmár-Nagy, Practical stability limits in turning, in: Proceedings of the ASME International Design Engineering Technical Conferences, no. DETC2009-87645, San Diego, CA, USA, 2009.

[17] K. Ahmadi, F. Ismail, Experimental investigation of process damping nonlinearity in machining chatter, International Journal of Machine Tools and Manufacture 50 (11) (2010) 1006-1014.

[18] K. Ahmadi, F. Ismail, Investigation of finite amplitude stability due to process damping in milling, in: Proceedings of the 5th CIRP Conference on High Performance Cutting, Vol. 1, Zurich, Switzerland, 2012, pp. 6065 . 
[19] Z. Dombóvári, G. Stépán, On the bistable zone of milling processes, Philosophical Transactions of the Royal Society A: Mathematical, Physical and Engineering Sciences 373 (2015) 20140409 (17 pages)

[20] G. Stépán, Retarded dynamical systems, Longman, Harlow, 1989.

[21] J. Hale, Theory of Functional Differential Equations, Springer, New York, 1977.

[22] B. D. Hassard, N. D. Kazarinoff, Y.-H. Wan, Theory and Applications of Hopf Bifurcation, London Mathematical Society Lecture Note Series 41 Cambridge, 1981.

[23] J. Guckenheimer, P. Holmes, Nonlinear Oscillations, Dynamical Systems, and Bifurcations of Vector Fields, Springer, New York, 1983.

[24] Y. A. Kuznetsov, Elements of Applied Bifurcation Theory, Springer, New York, 1998.

[25] S. A. Campbell, Calculating centre manifolds for delay differential equations using maple, in: B. Balachandran, T. Kalmar-Nagy, D. E. Gilsinn (Eds.), Delay Differential Equations, Springer, New York, 2009, pp. 221244.

[26] A. H. Nayfeh, D. T. Mook, Nonlinear Oscillations, Wiley, New York, 1979.

[27] E. Wesson, R. H. Rand, Hopf bifurcations in delayed rock-paper-scissors replicator dynamics, Dynamic Games and Applications 6 (1) (2016) 139156.

[28] P. Subramanian, R. I. Sujith, P. Wahi, Subcritical bifurcation and bistability in thermoacoustic systems, Journal of Fluid Mechanics 715 (2013) 210-238.

[29] M. Davidow, B. Shayak, R. H. Rand, Analysis of a remarkable singularity in a nonlinear DDE, Nonlinear Dynamics 90 (1) (2017) 317-323.

[30] A. H. Nayfeh, Order reduction of retarded nonlinear systems - the method of multiple scales versus center-manifold reduction, Nonlinear Dynamics 51 (4) (2008) 483-500.

[31] K. Nandakumar, P. Wahi, A. Chatterjee, Infinite dimensional slow modulations in a well known delayed model for cutting tool vibrations, Nonlinear Dynamics 62 (4) (2010) 705-716.

[32] G. Habib, G. Kerschen, G. Stépán, Chatter mitigation using the nonlinear tuned vibration absorber, International Journal of Non-Linear Mechanics 91 (2016) 103-112.

[33] N. Krylov, N. Bogoliubov, Introduction to Non-linear Mechanics, Princeton University Press, Princeton, 1947.

[34] J. Hale, Averaging methods for differential equations with retarded arguments and a small parameter, Journal of Differential Equations 2 (1) (1966) 57-73.

[35] J. Hale, S. M. V. Lunel, Averaging in infinite dimensions, Journal of Integral Equations and Applications 2 (4) (1990) 463-494.

[36] B. Lehman, S. P. Weibel, Fundamental theorems of averaging for functional differential equations, Journal of Differential Equations 152 (1) (1999) 160-190.

[37] L. Ng, R. H. Rand, Bifurcations in a Mathieu equation with cubic nonlinearities, Chaos, Solitons and Fractals 14 (2) (2002) 173-181.

[38] P. Wahi, A. Chatterjee, Averaging oscillations with small fractional damping and delayed terms, Nonlinear Dynamics 38 (1) (2004) 3-22.

[39] T. M. Morrison, R. H. Rand, 2:1 resonance in the delayed nonlinear Mathieu equation, Nonlinear Dynamics 50 (1-2) (2007) 341-352.

[40] J. A. Sanders, F. Verhulst, J. Murdock, Averaging Methods in Nonlinear Dynamical Systems, Springer, New York, 2007.

[41] T. Sari, Averaging for ordinary differential equations and functional differential equations, in: I. van den Berg, V. Neves (Eds.), The Strength of Nonstandard Analysis, Springer, Wien, 2007, pp. 286-305.

[42] R. H. Rand, Lecture Notes in Nonlinear Vibrations, Published on-line by The Internet-First University Press, 2012.

[43] G. Stépán, Z. Dombóvári, J. Muñoa, Identification of cutting force characteristics based on chatter experiments, CIRP Annals - Manufacturing Technology 60 (1) (2011) 113-116.

[44] F. W. Taylor, On the art of cutting metals, American Society of Mechanical Engineers, New York, 1907.

[45] W. J. Endres, M. Loo, Modeling cutting process nonlinearity for stability analysis - application to tooling selection for valve-seat machining, in: Proceedings of the 5th CIRP International Workshop on Modeling of Machining, West Lafayette, IN, USA, 2002, pp. 71-82.

[46] T. Insperger, G. Stépán, Semi-Discretization for Time-Delay Systems Stability and Engineering Applications, Springer, New York, 2011.

[47] R. Szalai, G. Stépán, S. J. Hogan, Global dynamics of low immersion high-speed milling, Chaos 14 (4) (2004) 1069-1077.

[48] Z. Dombóvári, D. A. Barton, R. E. Wilson, G. Stépán, On the global dynamics of chatter in the orthogonal cutting model, International Journal of Non-Linear Mechanics 46 (1) (2011) 330-338.

[49] T. Kalmár-Nagy, Delay-differential models of cutting tool dynamics with nonlinear and mode-coupling effects, Ph.D. thesis, Faculty of the Graduate School of Cornell University (2002).

[50] G. Stepan, D. Hajdu, A. Iglesias, D. Takacs, Z. Dombovari, Ultimate capability of variable pitch milling cutters, CIRP Annals - Manufacturing Technology 67 (1) (2018) 373-376.

[51] K. Engelborghs, T. Luzyanina, D. Roose, Numerical bifurcation analysis of delay differential equations using DDE-BIFTOOL, ACM Transactions on Mathematical Software 28 (1) (2002) 1-21.

[52] P. Wahi, Personal communication.

[53] T. G. Molnár, T. Insperger, G. Stépán, Analytical estimations of limit cycle amplitude for delay-differential equations, Electronic Journal of Qualitative Theory of Differential Equations 2016 (77) (2016) 1-10. 